\title{
Observer-Based Event-triggered Adaptive Containment Control for Multiagent Systems With Prescribed performance
}

\author{
Rui Xu • Xin Wang • Yuhao Zhou
}

Received: date / Accepted: date

\begin{abstract}
This paper focuses on the problem of the observer-based event-triggered adaptive containment control for a class of nonlinear multiagent systems (MASs) with prescribed performance. First, the radial basis function neural networks (RBFNNs) are adopted to approximate the uncertain smooth nonlinear function, and the neural network-based state observer is designed to estimate the unmeasurable state. Besides, to reduce the control resource assumption and get a better balance between the system performance and network constraints, the switching threshold based event-triggered control strategy is introduced. Based on this, the novel distributed containment controller is designed by utilizing the adaptive backstepping technique and the dynamic surface control (DSC) technique to guarantee that the output of each follower converges to the convex hull formed by multileader. Moreover, the containment errors can be converged to the prescribed boundary and all signals in closed-loop system are semi-global uniformly ultimately bounded (SGUUB) as well. Finally, the simulation example is carried out to illustrate the efficiency of the proposed controller.
\end{abstract}

Keywords Adaptive Containment Control · Event-triggered · prescribed performance · State Observer

\section{Introduction}

Over the past decades, cooperative control of multiagent systems (MASs) has gained growing popularity on account of its extensive applications in various fields, such as sensor networks [1] [2] and marine vessels [3] [4] as well as spacecraft formation flying [5], to name just a few. According to the number of the leaders, cooperative control problem can be segmented into three categories including leaderless consensus control [6], leader-following consensus control [7] and the containment control [8]. The fundamental characteristic of the containment control is that the MASs are composed of multiple leaders and all followers converge to the convex hull limited by multileader. In view of this, quite a few researches on containment control are designed for linear multiagent systems [9-11].

As is known to all, nonlinear multiagent systems are more suitable for describing practical application scenarios than linear ones. However, the dynamics of nonlinear multiagent systems are often difficult to obtain, which means the traditional adaptive control method is not suitable any more. Recently, the approximate capacity of the neural network and the fuzzy logic system (FLS) have shed some insightful light on the investigations of the adaptive control problem for nonlinear multiagent systems. Mei et al. [12] applied the universal approximation property of radial basis function neural

R. Xu, X. Wang( $(\varangle)$ and Y. Zhou

Chongqing Key Laboratory of Nonlinear Circuits and Intelligent Information Processing, College of Electronic and Information Engineering, Southwest University, Chongqing, 400044, P.R. China

E-mail: xinwangswu@163.com 
networks (RBFNNs) to tackle the obstacle of uncertain nonlinearities and proposed the NN-based distributed adaptive containment control scheme for second-order MASs with unknown nonlinear dynamics. Yoo [13] extended this approach to cope with consensus problems in high-order nonlinear strict-feedback multiagent systems under a directed graph topology, which can better be applied to the engineering systems. Besides, Wang and Tong [14] utilized FLSs to identify the unknown nonlinear functions and cope with the containment control problem for full-state-constrained nonlinear strict-feedback systems. However, the results described in [12-14] are based upon a quite ideal situation that the states of all followers are measurable, while it is inevitable that some states can not be obtained directly because of economic constraints or technological limitations. In this case, Tong and $\mathrm{Li}$ [15] introduced the fuzzy adaptive observer to estimate unmeasured states for single-input and single-output (SISO) nonlinear systems. Subsequently, an adaptive fuzzy backstepping dynamic surface control (DSC) method was introduced for multiple-input and multiple-output (MIMO) nonlinear systems in [16]. Note that, the DSC technique shares the merit in overcoming the "explosion of complexity" issue inherent in each adaptive backstepping design step. The general idea of this technique is to introduce the first-order filter in each process of the backstepping design procedure.

Recently, as system performance has become significantly important for practical systems, a number of control schemes with prescribed performance, especially coordinated transformation-based prescribed performance, become major concerns as open for nonlinear multiagent systems. [17-20]. The primary objective of prescribed performance is to guarantee that the control errors gather to the predefined performance bounds along with the convergence rate. In [20], the state feedback control approach is established to address the trouble of unknown pure-feedback nonlinear systems. Tong and $\mathrm{Li}$ [17] proposed the output feedback prescribed performance control method for SISO switched nonstrict-feedback nonlinear systems. The authors in [19] focused on the nonlinear large-scale systems consisting of immeasurable states and unknown time-varying delays. Ni et al. [21] investigated the fixed-time recurrent neural network control for uncertain strict-feedback nonlinear systems in the presence of unknown dead-zone output. More recently, by using FLSs, a quantized cooperative control strategy with the prespecified performance approach for MASs with unknown gains and input quantization was developed in [22]. Wang et al. [23] dealt with the issue of adaptive fuzzy containment control for nonlinear MASs with prescribed performance, unknown disturbance and unknown dead zone. However, the aforementioned results are time-triggered control, which will result in unnecessary resource consumption due to the continuously information transmission through the communication topology.

It should be mentioned that the research of event-triggered control schemes for nonlinear systems is of extraordinary significance as it can further improve the control efficiency as well as decrease heavy communication burden and cost than classical time-triggered strategy [24]. Xing et al. [25] put forward the original event-triggered control method named the switching threshold strategy based on the framework of the fixed threshold strategy [26] and the relative threshold strategy [27]. The proposed adaptive controller is able to compensate for the measurement errors so that the input-tostate stability (ISS) assumption is no longer needed. In [28], the event-triggered-based output feedback control mechanism was investigated for uncertain nonlinear systems. To decrease the communication channel bindwidth, the authors proposed the new 1-bit signal transmission rule which has been proved to be effective. Ma et al. [29] designed the observer-based adaptive event-triggered control method for the stochastic nonlinear systems along with full state constraints and actuator faults. In order to obtain accurate tracking performance, it is essential to extend the event-triggered control from nonlinear systems to MASs. Consequently, Zhang et al. [30] developed the event-triggered tracking control scheme for nonlinear MASs with unknown disturbances that can be estimated by a disturbance observer. The problem of distributed adaptive output control for uncertain nonlinear MASs with unmeasured states and uncertain control gains was addressed, and the Nussbaum function is used to compensate for the uncertain control gains in [31]. Besides, Qiu et al. [32] applied the fixed threshold event-triggered control mechanism to pure-feedback nonlinear systems with prescribed performance and unavailable system states. It is worth noting that the problems of event-triggered adaptive containment control for nonlinear MASs with immeasurable states and predefined performance are still to be studied.

Motivated by the analysis mentioned above, this paper is concerned with the observer-based eventtriggered adaptive containment control problem for a class of high-order uncertain nonlinear multiagent systems in strict-feedback form with prescribed performance. RBFNNs are utilized to approximate the 
unknown nonlinear function of the system dynamic, as well as the NN-based observer is applied to estimate the unavailable states. Combined with the Lyapunov stability theorem and backstepping techniques, the novel distributed adaptive containment control approach is proposed. The major contributions of this paper can be stated as follows: 1) By means of extending the switching threshold event-triggered strategy to the containment control of MASs, not only the communication burden has been alleviated but also the system performance can be ensured. In addition, the proposed control scheme can achieve the containment control objective. 2) Under the framework of prescribed performance control, the containment errors can converge to a predefined arbitrarily small compact set with rapid convergence speed and high accuracy. 3) The proposed control method can overcome the differentiation explosion by using the DSC technique, and thus, the designed controllers become more effective than conventional adaptive backstepping controllers, the containment control also gains superior performance. All variables of the MASs are semi-global uniformly ultimately bounded (SGUUB) as well.

The rest of this paper is described as follows. In Section II, the system formulation and preliminary knowledge are given. The controller design and stability analysis are shown in Section III. The effectiveness of this proposed strategy is verified by the simulation results in Section IV. Section V finally draws the conclusion.

\section{Problem Formulation and Preliminaries}

\subsection{Graph Theory}

The exchange of information among agents can be expressed as the directed graph $\mathcal{G}=(\mathcal{V}, \aleph, \mathcal{A})$, with the set of vertexes $\mathcal{V}=\{1,2, \ldots, N, N+1, \ldots, N+M\}$ and the set of edges $\aleph=\{(i, j) \mid i, j \in$ $\mathcal{V}, j \neq i\}$. The followers are labeled as $1, \ldots, N$, while " $N+1, \ldots, N+M$ " represent the leader. $\mathcal{A}=$ $\left[a_{i, j}\right] \in \mathbb{R}^{(N+M) \times(N+M)}$ stands for the adjacency matrix, where $(i, j) \in \aleph$ means that there exists an information flow from agent $j$ to agent $i$, and $a_{i, j}=1$ when $(i, j) \in \aleph$, otherwise $a_{i, j}=0$. The neighbors set of nodes $i$ is defined as $\mathcal{N}_{i}=\{j \mid(i, j) \in \aleph\}$. Let $\mathcal{L}=\left[l_{i, j}\right] \in \mathbb{R}^{(N+M) \times(N+M)}$ be the Laplacian matrix, and $l_{i, j}=-a_{i, j}$ for all $j \neq i$, otherwise, $l_{i, j}=\sum_{j \in \mathcal{N}_{i}} a_{i, j}$. The Laplacian matrix equals to $\mathcal{L}=\mathcal{D}-\mathcal{A}$, where $\mathcal{D}=\operatorname{diag}\left\{d_{1}, \ldots, d_{N+M}\right\}$ with $d_{i}=\sum_{j \in \mathcal{N}_{i}} a_{i, j}$ denotes the degree matrix of the digraph $\mathcal{G}$. It is assumed that each follower owns at least one neighbor, while leaders own none. Then, the Laplacian $\mathcal{L}$ of the digraph $\mathcal{G}$ can be partitioned into

$$
\mathcal{L}=\left[\begin{array}{cc}
\mathcal{L}_{1} & \mathcal{L}_{2} \\
0_{M \times N} & 0_{M \times M}
\end{array}\right]
$$

Assumption 1: For each follower, there is at least one leader that possesses a directed path to it.

Lemma 1 [33]: Based on Assumption 1, each eigenvalues of matrix $\mathcal{L}_{1}$ has a positive real part. Each row of $-\mathcal{L}_{1}^{-1} \mathcal{L}_{2}$ has a sum that equals to 1 , and each entry of $-\mathcal{L}_{1}^{-1} \mathcal{L}_{2}$ is nonnegative.

Assumption 2: The outputs of the leaders $y_{l, r}(l=N+1, \ldots, N+M)$ are adequately smooth function of t, and $y_{l, r}, \dot{y}_{l, r}, \ddot{y}_{l, r}$ are bounded, i.e.,there exists a positive constant $\varpi$, such that

$$
\left\|y_{l, r}\right\|^{2}+\left\|\dot{y}_{l, r}\right\|^{2}+\left\|\ddot{y}_{l, r}\right\|^{2} \leq \varpi
$$

Definition 1 [13]: A set $\mathfrak{C}$ is convex, if for any $X_{1}, X_{2} \in \mathfrak{C}$ and any $\theta \in[0,1], \theta X_{1}+(1-\theta) X_{2} \in \mathfrak{C}$. The convex hull of a set $\mathfrak{C}$, denoted $\mathfrak{C o}(X)\left(X=X_{1}, \ldots, X_{n}\right)$, is the smallest convex set that contains all points in $X$. Concretely, $\mathfrak{C o}(X)=\left\{\sum_{k=1}^{n} \theta_{k} X_{k} \mid X_{k} \in X, \theta_{k}>0, \sum_{k=1}^{n} \theta_{k}=1\right\}$.

\subsection{Model Formulation}

Consider a strict-feedback nonlinear multiagent system composed of $\mathrm{N}$ agents. The dynamics of the $i$ th $(i=1, \ldots, \mathrm{N})$ agents are described as follows:

$$
\begin{aligned}
& \dot{x}_{i, j}=x_{i, j+1}+f_{i, j}\left(\bar{x}_{i, j}\right), \quad j=1,2, \ldots, n-1 \\
& \dot{x}_{i, n}=u_{i}+f_{i, n}\left(\bar{x}_{i, n}\right) \\
& y_{i}=x_{i, 1}, \quad i=1,2, \ldots, N
\end{aligned}
$$


where $\bar{x}_{i, m}=\left[x_{i, 1}, x_{i, 2}, \ldots, x_{i, m}\right]^{\mathrm{T}} \in \mathbb{R}^{m}$ are state vectors. $y_{i} \in \mathbb{R}$ and $u_{i}(t) \in \mathbb{R}$ are the system output and the control input, respectively. $f_{i, j}(\cdot)$ denotes the unknown smooth nonlinear function. In this paper, it is assumed that only the output variables $y_{i}$ are available, while other states are unmeasurable during the controller design procedure.

Rewrite system (1) as:

$$
\begin{aligned}
& \dot{\bar{x}}_{i, n}=K_{i} \bar{x}_{i, n}+A_{i} y_{i}+\sum_{j=1}^{n} B_{i, j} f_{i, j}\left(\bar{x}_{i, j}\right)+C_{i} u_{i} \\
& y_{i}=D_{i}^{T} \bar{x}_{i, n}, \quad i=1,2, \ldots, N
\end{aligned}
$$

where

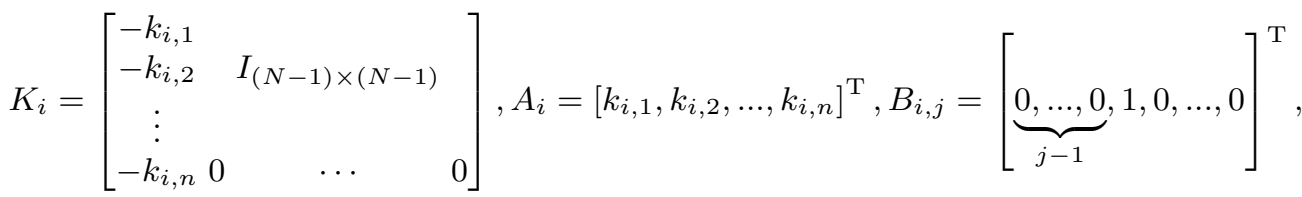

$$
\begin{aligned}
& C_{i}=[0, \ldots, 0,1]^{\mathrm{T}}, \quad D_{i}=[1,0, \ldots, 0]^{\mathrm{T}}
\end{aligned}
$$

The vector $A_{i}$ is selected so that $K_{i}$ is a strict Hurwitz matrix, thus, given any positive definite matrix $Q_{i}$ with $Q_{i}=Q_{i}^{\mathrm{T}}>0$, there is a positive symmetric matrix $P_{i}$ satisfying

$$
K_{i}^{\mathrm{T}} P_{i}+P_{i}^{\mathrm{T}} K_{i}=-Q_{i}
$$

\subsection{RBFNN and State-Observer Design}

The universal approximation property of RBFNNs are used to approximate an unknown smooth function $\Psi(X): R^{n} \rightarrow R^{m}$ defined on a compact set $\Omega_{x}$, there exists an RBFNN as:

$$
\Psi(X)=W^{* \mathrm{~T}} S(X)
$$

where $X \in \Omega_{x} \subset \mathbb{R}^{n}$ is the input vector, $W^{*} \in \mathbb{R}^{l \times m}$ is the ideal weight matrix, $l>1$ represents the number of neurons, $S(X)=\left[S_{1}(X), S_{2}(X), \ldots, S_{l}(X)\right]^{\mathrm{T}}$ is the Gaussian basis function vectors, and $S_{i}(X)=\exp \left[-\left(X-\varrho_{i}\right)^{\mathrm{T}}\left(X-\varrho_{i}\right) / \varsigma_{i}^{2}\right]$ for $i=1,2, \ldots, l$, where $\varrho_{i}=\left[\varrho_{i, 1}, \varrho_{i, 2}, \ldots, \varrho_{i, n}\right]^{\mathrm{T}}, i=1,2, \ldots, l$ denote the center of the receptive field, $\varsigma_{i}$ represents the width of the Gaussian function.

As is known to all, RBFNN can approximate any continues nonlinear function to a desired accuracy on a compact space. On account of the excellent ability of RBFNNs, the unknown smooth function $F_{i, j}\left(\bar{x}_{i, j}\right) \in \mathbb{R}^{m}$ can be approximated as:

$$
\hat{f}_{i, j}\left(\bar{x}_{i, j} \mid \hat{W}_{i, j}\right)=\hat{W}_{i, j}^{\mathrm{T}} S_{i, j}\left(\bar{x}_{i, j}\right)
$$

where $\hat{W}_{i, j}$ represents the estimation of the optimal weight matrix $W_{i, j}^{*}$.

Let $\hat{\bar{x}}_{i, j}=\left[\hat{x}_{i, 1}, \ldots, \hat{x}_{i, j}\right]^{\mathrm{T}}$ denote the estimation of the state vector $\bar{x}_{i, j}$, One has

$$
\hat{f}_{i, j}\left(\hat{\bar{x}}_{i, j} \mid \hat{W}_{i, j}\right)=\hat{W}_{i, j}^{\mathrm{T}} S_{i, j}\left(\hat{\bar{x}}_{i, j}\right)
$$

The optimal weight matrix $W_{i, j}^{*}$ is expressed as:

$$
W_{i, j}^{*}:=\arg \min _{\hat{W}_{i, j} \in \tilde{\Xi}_{i, j} \in \mathbb{R}^{l \times m}}\left\{\sup _{\substack{\hat{x}_{i, j} \in \hat{\Xi}_{i, j} \\ \bar{x}_{i, j} \in \Xi_{i, j}}}\left\|\hat{f}_{i, j}\left(\hat{\bar{x}}_{i, j} \mid \hat{W}_{i, j}\right)-f_{i, j}\left(\bar{x}_{i, j}\right)\right\|\right\}
$$

where $\Xi_{i, j}, \hat{\Xi}_{i, j}$ and $\tilde{\Xi}_{i, j}$ are compact sets for $\bar{x}_{i, j}, \hat{\bar{x}}_{i, j}$ and $\hat{W}_{i, j}$, respectively. 
Defining the corresponding minimal approximation errors $\lambda_{i, j}$ and the approximation errors $\mu_{i, j}$ respectively, as

$$
\begin{aligned}
& \lambda_{i, j}=f_{i, j}\left(\bar{x}_{i, j}\right)-\hat{f}_{i, j}\left(\hat{\bar{x}}_{i, j} \mid W_{i, j}^{*}\right) \\
& \mu_{i, j}=f_{i, j}\left(\bar{x}_{i, j}\right)-\hat{f}_{i, j}\left(\hat{\bar{x}}_{i, j} \mid \hat{W}_{i, j}\right)
\end{aligned}
$$

Assumption 3: The corresponding minimal approximation errors $\lambda_{i, j}$ and the approximation errors $\mu_{i, j}$ are bounded, i.e., existing the constant $\lambda_{i, j}^{0}>0, \mu_{i, j}^{0}>0$ such that $\left\|\lambda_{i, j}\right\|<\lambda_{i, j}^{0},\left\|\mu_{i, j}\right\|<\mu_{i, j}^{0}$. Defining $\eta_{i, j}=\lambda_{i, j}-\mu_{i, j}, \eta_{i, j}^{0}=\lambda_{i, j}^{0}+\mu_{i, j}^{0}$, then we have $\left\|\eta_{i, j}\right\| \leq \lambda_{i, j}^{0}+\mu_{i, j}^{0}=\eta_{i, j}^{0}, i=1, \ldots, N, j=$ $1, \ldots, n$.

Since the states of each follower in system (1) except outputs are unavailable in the control design, the NN-based state observers is constructed to estimate the unavailable states:

$$
\begin{aligned}
& \dot{\overline{\bar{x}}}_{i, n}=K_{i} \hat{\bar{x}}_{i, n}+A_{i} y_{i}+\sum_{j=1}^{n} B_{i, j} \hat{f}_{i, j}\left(\hat{\bar{x}}_{i, j} \mid \hat{W}_{i, j}\right)+C_{i} u_{i} \\
& y_{i}=D_{i}^{T} \hat{\bar{x}}_{i, n}, \quad i=1,2, \ldots, N
\end{aligned}
$$

Define the observer error as $e_{i}=\left[e_{i, 1}, e_{i, 2}, \ldots, e_{i, n}\right]^{\mathrm{T}}=\bar{x}_{i, n}-\hat{\bar{x}}_{i, n}=\left[\left(x_{i, 1}-\hat{x}_{i, 1}\right)^{\mathrm{T}}, \ldots,\left(x_{i, n}-\right.\right.$ $\left.\left.\hat{x}_{i, n}\right)^{\mathrm{T}}\right]^{\mathrm{T}}$, then from $(2)$ and $(9)$, the observer error dynamic can be written as

$$
\begin{aligned}
e_{i} & =K_{i} e_{i}+\sum_{j=1}^{n} B_{i, j} f_{i, j}\left(\bar{x}_{i, j}\right)-\sum_{j=1}^{n} B_{i, j} \hat{f}_{i, j}\left(\hat{\bar{x}}_{i, j} \mid \hat{W}_{i, j}\right) \\
& =K_{i} e_{i}+\mu_{i}, \quad i=1, \ldots, N
\end{aligned}
$$

where $\mu_{i}=\left[\mu_{i, 1}, \ldots, \mu_{i, n}\right]^{\mathrm{T}}$

By combining (1), (9), and (10), one obtains:

$$
\begin{aligned}
& \dot{\hat{x}}_{i, j}=\hat{x}_{i, j+1}+k_{i, j}\left(x_{i, 1}-\hat{x}_{i, 1}\right)+\hat{f}_{i, j}\left(\hat{\bar{x}}_{i, j} \mid \hat{W}_{i, j}\right), \quad j=1,2, \ldots, n-1 \\
& \dot{\hat{x}}_{i, n}=u_{i}+k_{i, n}\left(x_{i, 1}-\hat{x}_{i, 1}\right)+\hat{f}_{i, n}\left(\hat{\bar{x}}_{i, n} \mid \hat{W}_{i, n}\right)
\end{aligned}
$$

\subsection{Event-triggered Design}

A switching threshold event-triggered control strategy is given below. The intermediate control function is defined as follows:

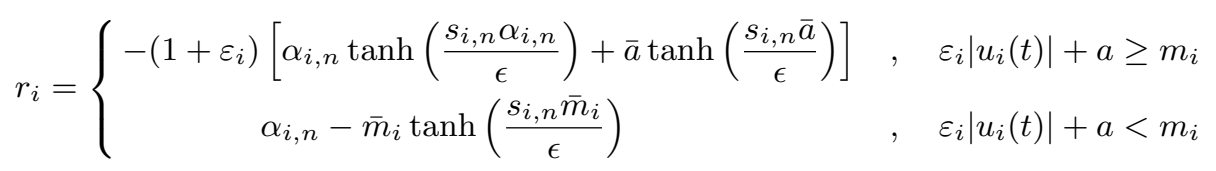

where $\alpha_{i, n}$ is virtual control function to be designed later.

Define the triggering event as

$$
\begin{aligned}
& u_{i}(t)=r_{i}\left(t_{i}^{k}\right) \quad \forall t \in\left[t_{i}^{k}, t_{i}^{k+1}\right) \\
& t_{i}^{k+1}=\inf \left\{t \in \mathbb{R} \mid \tau_{i}(t) \geq \max \left\{\varepsilon_{i}\left|u_{i}(t)\right|+a, m_{i}\right\}\right\}
\end{aligned}
$$

where $\tau_{i}(t)=r_{i}(t)-u_{i}(t)$ denotes the measurement error. $t_{i}^{k}, k \in z^{+}$is the update time, i.e., when (14) is triggered, the time will be recorded by $t_{i}^{k+1}$, and the control value will be marked as $u\left(t_{i}^{k+1}\right)$. $\varepsilon_{i}, m_{i}, \bar{m}_{i}>m_{i}, a$ and $\bar{a}>a /\left(1-\varepsilon_{i}\right)$ are positive design parameters, $0<\varepsilon_{i}<1$. During the period $t \in\left[t_{i}^{k}, t_{i}^{k+1}\right)$, the control signal holds as $r_{i}\left(t_{i}^{k}\right)$.

From (13) and (14), we have

$$
\tau_{i}(t)=\left\{\begin{array}{ccc}
\delta_{i, 1}(t) \varepsilon_{i} u_{i}(t)+\delta_{i, 2}(t) a & , & \varepsilon_{i}\left|u_{i}(t)\right|+a \geq m_{i} \\
\delta_{i, 3}(t) m_{i} & , & \varepsilon_{i}\left|u_{i}(t)\right|+a<m_{i}
\end{array}\right.
$$


where $\delta_{i, 1}(t), \delta_{i, 2}(t)$ and $\delta_{i, 3}(t)$ are continuous time-varying parameters with $\delta_{i, 1}\left(t_{i}^{k}\right)=\delta_{i, 2}\left(t_{i}^{k}\right)=$ $\delta_{i, 3}\left(t_{i}^{k}\right)=0, \delta_{i, 1}\left(t_{i}^{k+1}\right)= \pm 1, \delta_{i, 2}\left(t_{i}^{k+1}\right)= \pm 1, \delta_{i, 3}\left(t_{i}^{k+1}\right)= \pm 1,\left|\delta_{i, 1}(t)\right| \leq 1,\left|\delta_{i, 2}(t)\right| \leq 1,\left|\delta_{i, 3}(t)\right| \leq$ $1, \forall t \in\left[t_{i}^{k}, t_{i}^{k+1}\right)$. Then, one has

$$
u_{i}(t)=\left\{\begin{array}{ccc}
\frac{r_{i}}{1+\delta_{i, 1}(t) \varepsilon_{i}}-\frac{\delta_{i, 2}(t) a}{1+\delta_{i, 1}(t) \varepsilon_{i}} & , & \varepsilon_{i}\left|u_{i}(t)\right|+a \geq m_{i} \\
r_{i}-\delta_{i, 3}(t) m_{i} & , & \varepsilon_{i}\left|u_{i}(t)\right|+a<m_{i}
\end{array}\right.
$$

Remark 1: This switching threshold-based controller has the advantages of the fixed threshold strategy and the relative threshold strategy. As there is a high magnitude of control signal $u_{i}(t)$, the relative threshold strategy is chosen to obtain longer update intervals; while when $u_{i}(t)$ is approaching to zero, the fixed threshold strategy is applied to obtain better system performance. The Zeno behavior can be avoided as well.

Lemma 2 [25]: The hyperbolic tangent function $\tanh (\cdot)$ satisfies the following property:

$$
0 \leq|\iota|-\iota \tanh \frac{\iota}{\epsilon} \leq 0.2785 \epsilon
$$

where $\epsilon$ is an arbitrary positive constant, and $\iota \in \mathbb{R}$.

\subsection{Prescribed Performance}

According to [20], the prescribed performance is accomplished by ensuring that the containment error surfaces $v_{i, 1}=\sum_{j=1}^{N} a_{i, j}\left(y_{i}-y_{j}\right)+\sum_{l=N+1}^{N+M} a_{i, l}\left(y_{i}-y_{l, r}\right)$ evolve strictly within predefined decaying bounds as follows:

$$
-\eta_{i \min } \omega_{i}(t)<v_{i, 1}(t)<\eta_{i \max } \omega_{i}(t), \quad \forall t \geq 0
$$

where $\eta_{i \text { min }}$ and $\eta_{i \max }$ are positive parameters. The prescribed performance function $\omega_{i}(t)=\left(\omega_{i, 0}-\right.$ $\left.\omega_{i, \infty}\right) e^{-\zeta_{i} t}+\omega_{i, \infty}$ is a strictly decreasing smooth function satisfying $\lim _{t \rightarrow \infty} \omega_{i}(t)=\omega_{i, \infty}>0, \omega_{i, 0}=$ $\omega_{i}(0)>\omega_{i, \infty}, \zeta_{i}>0$ such that $-\eta_{i \min } \omega_{i}(0)<v_{i, 1}(0)<\eta_{i \max } \omega_{i}(0)$, and we can obtain $\left|v_{i, 1}(t)\right|<$ $\max \left\{\eta_{i \min } \omega_{i}(0), \eta_{i \max } \omega_{i}(0)\right\}$.

To achieve the desired performance, the constrained containment error is converted into an equivalent unconstrained one. Letting

$$
v_{i, 1}(t)=\omega_{i}(t) \Theta_{i}\left(\beta_{i}(t)\right), \quad \forall t \geq 0
$$

where $\beta_{i}$ is the converted error, and the smooth function $\Theta_{i}\left(\beta_{i}\right)$ is strictly monotonic increasing, and

$$
\Theta_{i}\left(\beta_{i}\right)=\frac{\eta_{i \max } e^{\beta_{i}}-\eta_{i \min } e^{-\beta_{i}}}{e^{\beta_{i}}+e^{-\beta_{i}}}
$$

The inverse function can be represented as:

$$
\beta_{i}(t)=\Theta_{i}^{-1}\left(\frac{v_{i, 1}(t)}{\omega_{i}(t)}\right)=\frac{1}{2} \ln \frac{\Theta_{i}+\eta_{i \min }}{\eta_{i \max }-\Theta_{i}}
$$

and

$$
\dot{\beta}_{i}(t)=\pi_{i}\left(\dot{v}_{i, 1}-\frac{\dot{\omega}_{i} v_{i, 1}}{\omega_{i}}\right)
$$

where $\pi_{i}=\frac{1}{2 \omega_{i}}\left[\frac{1}{\Theta_{i}+\eta_{i \min }}-\frac{1}{\Theta_{i}-\eta_{i \max }}\right]$

Define the following state transformation:

$$
s_{i, 1}=\beta_{i}(t)-\frac{1}{2} \ln \frac{\eta_{i \min }}{\eta_{i \max }}
$$


the transformation state dynamics are

$$
\dot{s}_{i, 1}=\pi_{i}\left(\dot{v}_{i, 1}-\frac{\dot{\omega}_{i} v_{i, 1}}{\omega_{i}}\right)
$$

Similar to [20], it is obvious that $v_{i, 1}$ satisfies the prescribed performance expressed by (17) when $s_{i, 1}$ is bounded.

Remark 2: Different form the control strategy in [19] and [22] which can not be applied to the MASs with multileader, this paper attempts to deal with the containment control problems for MASs. Moreover, the unmeasured states and the switching threshold-based event-triggered strategy are incorporated in this work.

\section{Controller Design and Stability Analysis}

In this section, an adaptive neural network-based controller and parameter adaptive laws are presented by the backstepping technique and DSC technique. All signals of the closed-loop system (1) are SGUUB, and the containment errors $v_{i, 1}$ are as small as possible.

To design n-step adaptive backstepping consensus controller, the coordinate transformations are defined as:

$$
\begin{aligned}
& s_{i, j}=\hat{x}_{i, j}-\nu_{i, j} \\
& z_{i, j}=\nu_{i, j}-\alpha_{i, j-1}, \quad i=1, \ldots, N, j=2, \ldots, n
\end{aligned}
$$

where $s_{i, j}$ is an error surface, $\nu_{i, j}$ is a filtered virtual control, which is obtained through a first-order filter on virtual control function $\alpha_{i, j-1}$, and $z_{i, j}$ is the output error of the first-order filter.

Lemma 3 [23]: Letting $y_{i}=\left[y_{1}, \ldots, y_{N}\right]^{\mathrm{T}}$, and $s_{*, 1}=\left[s_{1,1}, \ldots, s_{N, 1}\right]^{\mathrm{T}}$. Then, the following inequality holds:

$$
\left\|y_{i}+\mathcal{L}_{1}^{-1} \mathcal{L}_{2} y_{l, r}\right\| \leq\left\|s_{*, 1}\right\| /\left\|\bar{\sigma}\left(\mathcal{L}_{1}\right)\right\|
$$

where $\bar{\sigma}$ is the minimum singular value of $\mathcal{L}_{1}$

Step 1: Combining (1), (7), (10) with (23), the time derivative of $v_{i, 1}=\sum_{j=1}^{N} a_{i, j}\left(y_{i}-y_{j}\right)+$ $\sum_{l=N+1}^{N+M} a_{i, l}\left(y_{i}-y_{l, r}\right)$ is

$$
\begin{aligned}
\dot{v}_{i, 1} & =\sum_{j=1}^{N} a_{i, j}\left(\dot{y}_{i}-\dot{y}_{j}\right)+\sum_{l=N+1}^{N+M} a_{i, l}\left(\dot{y}_{i}-\dot{y}_{l, r}\right) \\
& =d_{i} \dot{x}_{i, 1}-\sum_{j=1}^{N} a_{i, j} \dot{x}_{j, 1}-\sum_{l=N+1}^{N+M} a_{i, l} \dot{y}_{l, r} \\
& =d_{i}\left(\hat{x}_{i, 2}+f_{i, 1}\left(x_{i, 1}\right)+e_{i, 2}\right)-\sum_{j=1}^{N} a_{i, j}\left(\hat{x}_{j, 2}+f_{j, 1}\left(x_{j, 1}\right)+e_{j, 2}\right)-\sum_{l=N+1}^{N+M} a_{i, l} \dot{y}_{l, r} \\
& =d_{i}\left(\hat{x}_{i, 2}+\hat{W}_{i, 1}^{\mathrm{T}} S_{i, 1}\left(\hat{x}_{i, 1}\right)+\tilde{W}_{i, 1}^{\mathrm{T}} S_{i, 1}\left(\hat{x}_{i, 1}\right)+\lambda_{i, 1}+e_{i, 2}\right) \\
& -\sum_{j=1}^{N} a_{i, j}\left(\hat{x}_{j, 2}+\hat{W}_{j, 1}^{\mathrm{T}} S_{j, 1}\left(\hat{x}_{j, 1}\right)+\tilde{W}_{j, 1}^{\mathrm{T}} S_{j, 1}\left(\hat{x}_{j, 1}\right)+\lambda_{j, 1}+e_{j, 2}\right)-\sum_{l=N+1}^{N+M} a_{i, l} \dot{y}_{l, r} \\
& =d_{i}\left(\alpha_{i, 1}+z_{i, 2}+s_{i, 2}+\hat{W}_{i, 1}^{\mathrm{T}} S_{i, 1}\left(\hat{x}_{i, 1}\right)+\tilde{W}_{i, 1}^{\mathrm{T}} S_{i, 1}\left(\hat{x}_{i, 1}\right)+\lambda_{i, 1}+e_{i, 2}\right) \\
& -\sum_{j=1}^{N} a_{i, j}\left(\hat{x}_{j, 2}+\hat{W}_{j, 1}^{\mathrm{T}} S_{j, 1}\left(\hat{x}_{j, 1}\right)+\tilde{W}_{j, 1}^{\mathrm{T}} S_{j, 1}\left(\hat{x}_{j, 1}\right)+\lambda_{j, 1}+e_{j, 2}\right)-\sum_{l=N+1}^{N+M} a_{i, l} \dot{y}_{l, r}
\end{aligned}
$$

where $\tilde{W}_{i, j}=W_{i, j}^{*}-\hat{W}_{i, j}, j=1, \ldots, n$. 
Consider the following Lyapunov function candidate

$$
V_{i, 1}=e_{i}^{\mathrm{T}} P_{i} e_{i}+\frac{1}{2} s_{i, 1}^{2}+\frac{1}{2 \gamma_{i, 1}} \tilde{W}_{i, 1}^{\mathrm{T}} \tilde{W}_{i, 1}+\sum_{j=1}^{N} \frac{a_{i, j}}{2 \chi_{j, 1}} \tilde{W}_{j, 1}^{\mathrm{T}} \tilde{W}_{j, 1}
$$

where $\gamma_{i, 1}, \chi_{j, 1}$ are both positive design constants.

The time derivative of $V_{i, 1}$ along (3), (10) and (22) is

$$
\dot{V}_{i, 1}=-e_{i}^{\mathrm{T}} Q_{i} e_{i}+2 e_{i}^{\mathrm{T}} P_{i} \mu_{i}+s_{i, 1}^{\mathrm{T}} \pi_{i}\left(\dot{v}_{i, 1}-\frac{\dot{\omega}_{i} v_{i, 1}}{\omega_{i}}\right)-\frac{1}{\gamma_{i, 1}} \tilde{W}_{i, 1}^{\mathrm{T}} \dot{\hat{W}}_{i, 1}-\sum_{j=1}^{N} \frac{a_{i, j}}{\chi_{j, 1}} \tilde{W}_{j, 1}^{\mathrm{T}} \dot{\hat{W}}_{j, 1}
$$

Using the Young's inequality $2 a^{\mathrm{T}} b \leq\|a\|^{2}+\|b\|^{2}$, one has

$$
\begin{aligned}
2 e_{i}^{\mathrm{T}} P_{i} \mu_{i} & \leq\left\|e_{i}\right\|^{2}+\left\|P_{i}\right\|^{2}\left\|\mu_{i}^{0}\right\|^{2} \\
s_{i, 1}^{\mathrm{T}} \pi_{i}\left[d_{i}\left(\lambda_{i, 1}+e_{i, 2}\right)-\sum_{j=1}^{N} a_{i, j}\left(\lambda_{j, 1}+e_{j, 2}\right)\right] & \leq \pi_{i}^{2}\left\|s_{i, 1}\right\|^{2}+\frac{1}{2}\left\|\mathcal{L}_{1}\right\|_{F}^{2}\left\|\lambda_{i, 1}^{0}\right\|^{2}+\frac{1}{2}\left\|\mathcal{L}_{1}\right\|_{F}^{2}\left\|e_{i, 2}\right\|^{2} \\
& \leq \pi_{i}^{2}\left\|s_{i, 1}\right\|^{2}+\frac{1}{2}\left\|\mathcal{L}_{1}\right\|_{F}^{2}\left\|\lambda_{i, 1}^{0}\right\|^{2}+\frac{1}{2}\left\|\mathcal{L}_{1}\right\|_{F}^{2}\left\|e_{i}\right\|^{2}
\end{aligned}
$$

Substituting (24), (27), (28) into (26) results in

$$
\begin{aligned}
\dot{V}_{i, 1} & \leq-l_{\text {min }}\left\|e_{i}\right\|^{2}+\left\|P_{i}\right\|^{2}\left\|\mu_{i}^{0}\right\|^{2}+\frac{1}{2}\left\|\mathcal{L}_{1}\right\|_{F}^{2}\left\|\lambda_{i, 1}^{0}\right\|^{2}-\frac{1}{\gamma_{i, 1}} \tilde{W}_{i, 1}^{\mathrm{T}} \dot{\hat{W}}_{i, 1}-\sum_{j=1}^{N} \frac{a_{i, j}}{\chi_{j, 1}} \tilde{W}_{j, 1}^{\mathrm{T}} \dot{\hat{W}}_{j, 1} \\
& +s_{i, 1}^{\mathrm{T}} \pi_{i}\left[d_{i}\left(\alpha_{i, 1}+z_{i, 2}+s_{i, 2}+\hat{W}_{i, 1}^{\mathrm{T}} S_{i, 1}\left(\hat{x}_{i, 1}\right)+\tilde{W}_{i, 1}^{\mathrm{T}} S_{i, 1}\left(\hat{x}_{i, 1}\right)\right)-\pi_{i} s_{i, 1}\right. \\
& \left.-\sum_{j=1}^{N} a_{i, j}\left(\hat{x}_{j, 2}+\hat{W}_{j, 1}^{\mathrm{T}} S_{j, 1}\left(\hat{x}_{j, 1}\right)+\tilde{W}_{j, 1}^{\mathrm{T}} S_{j, 1}\left(\hat{x}_{j, 1}\right)\right)-\sum_{l=N+1}^{N+M} a_{i, l} \dot{y}_{l, r}-\frac{\dot{\omega}_{i} v_{i, 1}}{\omega_{i}}\right]
\end{aligned}
$$

where $l_{\min }=\min \left(\lambda_{\min }\left(Q_{i}\right)-1-\frac{1}{2}\left\|\mathcal{L}_{1}\right\|_{F}^{2}\right)$, and $\lambda_{\min }\left(Q_{i}\right)$ is the smallest eigenvalue of matrix $Q_{i}$.

Design the virtual control function $\alpha_{i, 1}$ and the parameter adaptive law $W_{i, 1}, W_{j, 1}$ as follows:

$$
\begin{aligned}
\alpha_{i, 1} & =\frac{1}{d_{i}}\left[-\frac{c_{i, 1}}{\pi_{i}} s_{i, 1}+\pi_{i} s_{i, 1}-\sum_{j=1}^{N} a_{i, j}\left(\hat{x}_{j, 2}+\hat{W}_{j, 1}^{\mathrm{T}} S_{j, 1}\left(\hat{x}_{j, 1}\right)\right)+\sum_{l=N+1}^{N+M} a_{i, l} \dot{y}_{l, r}+\frac{\dot{\omega}_{i} v_{i, 1}}{\omega_{i}}\right] \\
& -\hat{W}_{i, 1}^{\mathrm{T}} S_{i, 1}\left(\hat{x}_{i, 1}\right) \\
\dot{\hat{W}}_{i, 1} & =\gamma_{i, 1}\left(\pi_{i} d_{i} s_{i, 1} S_{i, 1}\left(\hat{x}_{i, 1}\right)-\sigma_{i, 1} \hat{W}_{i, 1}\right) \\
\dot{\hat{W}}_{j, 1} & =\chi_{j, 1}\left(\pi_{i} s_{i, 1} S_{j, 1}\left(\hat{x}_{j, 1}\right)-\varrho_{j, 1} \hat{W}_{j, 1}\right)
\end{aligned}
$$

where $c_{i, 1}>0, \sigma_{i, 1}>0$ and $\varrho_{j, 1}>0$ are design parameters.

By invoking (30) - (32), we can obtain

$$
\begin{aligned}
\dot{V}_{i, 1} & \leq-l_{m i n}\left\|e_{i}\right\|^{2}-c_{i, 1} s_{i, 1}^{2}+\pi_{i} d_{i} s_{i, 1}^{\mathrm{T}}\left(z_{i, 2}+s_{i, 2}\right)+\sigma_{i, 1} \tilde{W}_{i, 1}^{\mathrm{T}} \hat{W}_{i, 1} \\
& +\sum_{j=1}^{N} a_{i, j} \varrho_{j, 1} \tilde{W}_{j, 1}^{\mathrm{T}} \hat{W}_{j, 1}+\rho_{i, 1}
\end{aligned}
$$

where $\rho_{i, 1}=\left\|P_{i}\right\|^{2}\left\|\mu_{i}^{0}\right\|^{2}+\frac{1}{2}\|\mathcal{L}\|_{F}^{2}\left\|\lambda_{i, 1}^{0}\right\|^{2}$.

Define first-order filter with time constant $\tau_{i, j}$ as:

$$
\tau_{i, j} \dot{\nu}_{i, j}+\nu_{i, j}=\alpha_{i, j-1}, \quad \nu_{i, j}(0)=\alpha_{i, j-1}(0)
$$

as $\dot{z}_{i, j}=\dot{\nu}_{i, j}-\dot{\alpha}_{i, j-1}$, we obtain $\dot{z}_{i, j}=-\frac{z_{i, j}}{\tau_{i, j}}+M_{i, j}\left(s_{i, 1}, \ldots, s_{i, j}, z_{i, 2}, \ldots, z_{i, j}, \hat{W}_{i, 1}, \ldots, \hat{W}_{i, j}, y_{l, r}, \dot{y}_{l, r}, \ddot{y}_{l, r}\right)$ 
Step 2: from (5), (11), one obtains

$$
\dot{\hat{x}}_{i, 2}=\hat{x}_{i, 3}-k_{i, 2} \hat{x}_{i, 1}+k_{i, 2} x_{i, 1}+\hat{W}_{i, 2}^{\mathrm{T}} S_{i, 2}\left(\hat{\bar{x}}_{i, 2}\right)
$$

Consider the following Lyapunov function candidate

$$
V_{i, 2}=V_{i, 1}+\frac{1}{2} s_{i, 2}^{2}+\frac{1}{2} z_{i, 2}^{2}+\frac{1}{2 \gamma_{i, 2}} \tilde{W}_{i, 2}^{\mathrm{T}} \tilde{W}_{i, 2}
$$

where $\gamma_{i, 2}$ is a positive design constant.

The time derivative of $V_{i, 2}$ along (7), (8), (23), (33) - (35) is

$$
\begin{aligned}
\dot{V}_{i, 2} & =-l_{\text {min }}\left\|e_{i}\right\|^{2}-c_{i, 1} s_{i, 1}^{2}+s_{i, 1}^{\mathrm{T}} \pi_{i} d_{i}\left(z_{i, 2}+s_{i, 2}\right)+\sigma_{i, 1} \tilde{W}_{i, 1}^{\mathrm{T}} \hat{W}_{i, 1}+\rho_{i, 1} \\
& +s_{i, 2}^{\mathrm{T}}\left(s_{i, 3}+z_{i, 3}+\alpha_{i, 2}-k_{i, 2} \hat{x}_{i, 1}+k_{i, 2} x_{i, 1}+\hat{W}_{i, 2}^{\mathrm{T}} S_{i, 2}\left(\hat{\bar{x}}_{i, 2}\right)+\tilde{W}_{i, 2}^{\mathrm{T}} S_{i, 2}\left(\hat{\bar{x}}_{i, 2}\right)-\tilde{W}_{i, 2}^{\mathrm{T}} S_{i, 2}\left(\hat{\bar{x}}_{i, 2}\right)-\dot{\nu}_{i, 2}\right) \\
& -\frac{1}{\tau_{i, 2}} z_{i, 2}^{2}+z_{i, 2} M_{i, 2}(\cdot)-\frac{1}{\gamma_{i, 2}} \tilde{W}_{i, 2}^{\mathrm{T}} \dot{\hat{W}}_{i, 2}+\sum_{j=1}^{N} a_{i, j} \varrho_{j, 1} \tilde{W}_{j, 1}^{\mathrm{T}} \hat{W}_{j, 1} \\
& =-l_{m i n}\left\|e_{i}\right\|^{2}-c_{i, 1} s_{i, 1}^{2}+s_{i, 1}^{\mathrm{T}} \pi_{i} d_{i}\left(z_{i, 2}+s_{i, 2}\right)+\sigma_{i, 1} \tilde{W}_{i, 1}^{\mathrm{T}} \hat{W}_{i, 1}+\rho_{i, 1} \\
& +s_{i, 2}^{\mathrm{T}}\left(s_{i, 3}+z_{i, 3}+\alpha_{i, 2}-k_{i, 2} \hat{x}_{i, 1}+k_{i, 2} x_{i, 1}+\hat{W}_{i, 2}^{\mathrm{T}} S_{i, 2}\left(\hat{\bar{x}}_{i, 2}\right)+\tilde{W}_{i, 2}^{\mathrm{T}} S_{i, 2}\left(\hat{\bar{x}}_{i, 2}\right)+\lambda_{i, 2}-\mu_{i, 2}-\dot{\nu}_{i, 2}\right) \\
& -\frac{1}{\tau_{i, 2}} z_{i, 2}^{2}+z_{i, 2} M_{i, 2}(\cdot)-\frac{1}{\gamma_{i, 2}} \tilde{W}_{i, 2}^{\mathrm{T}} \dot{\hat{W}}_{i, 2}+\sum_{j=1}^{N} a_{i, j} \varrho_{j, 1} \tilde{W}_{j, 1}^{\mathrm{T}} \hat{W}_{j, 1}
\end{aligned}
$$

According to Young's inequality,we have

$$
s_{i, j}\left(\lambda_{i, j}-\mu_{i, j}\right) \leq \frac{1}{2} s_{i, j}^{2}+\frac{1}{2}\left\|\eta_{i, j}^{0}\right\|^{2}
$$

By invoking (38), we can obtain

$$
\begin{aligned}
\dot{V}_{i, 2} & \leq-l_{\text {min }}\left\|e_{i}\right\|^{2}-c_{i, 1} s_{i, 1}^{2}+s_{i, 1}^{\mathrm{T}} \pi_{i} d_{i}\left(z_{i, 2}+s_{i, 2}\right)+\sigma_{i, 1} \tilde{W}_{i, 1}^{\mathrm{T}} \hat{W}_{i, 1} \\
& +s_{i, 2}^{\mathrm{T}}\left(s_{i, 3}+z_{i, 3}+\alpha_{i, 2}-k_{i, 2} \hat{x}_{i, 1}+k_{i, 2} x_{i, 1}+\hat{W}_{i, 2}^{\mathrm{T}} S_{i, 2}\left(\hat{\bar{x}}_{i, 2}\right)+\tilde{W}_{i, 2}^{\mathrm{T}} S_{i, 2}\left(\hat{\bar{x}}_{i, 2}\right)+\frac{1}{2} s_{i, 2}-\dot{\nu}_{i, 2}\right) \\
& -\frac{1}{\tau_{i, 2}} z_{i, 2}^{2}+z_{i, 2} M_{i, 2}(\cdot)-\frac{1}{\gamma_{i, 2}} \tilde{W}_{i, 2}^{\mathrm{T}} \dot{\hat{W}}_{i, 2}+\sum_{j=1}^{N} a_{i, j} \varrho_{j, 1} \tilde{W}_{j, 1}^{\mathrm{T}} \hat{W}_{j, 1}+\rho_{i, 1}+\frac{1}{2}\left\|\eta_{i, 2}^{0}\right\|^{2}
\end{aligned}
$$

Construct the virtual control function $\alpha_{i, 2}$ and the parameter adaptive law $W_{i, 2}$ as:

$$
\begin{aligned}
& \alpha_{i, 2}=-c_{i, 2} s_{i, 2}-\pi_{i} d_{i} s_{i, 1}-\frac{1}{2} s_{i, 2}+k_{i, 2} \hat{x}_{i, 1}-k_{i, 2} x_{i, 1}-\hat{W}_{i, 2}^{\mathrm{T}} S_{i, 2}\left(\hat{\bar{x}}_{i, 2}\right)+\dot{\nu}_{i, 2} \\
& \dot{\hat{W}}_{i, 2}=\gamma_{i, 2}\left(s_{i, 2} S_{i, 2}\left(\hat{\bar{x}}_{i, 2}\right)-\sigma_{i, 2} \hat{W}_{i, 2}\right)
\end{aligned}
$$

where $c_{i, 2}>0$ and $\sigma_{i, 2}>0$ are design parameters.

From (39),(40) and (41), one has

$$
\begin{aligned}
\dot{V}_{i, 2} & \leq-l_{\text {min }}\left\|e_{i}\right\|^{2}-c_{i, 1} s_{i, 1}^{2}+s_{i, 1}^{\mathrm{T}} \pi_{i} d_{i} z_{i, 2}-c_{i, 2} s_{i, 2}^{2}+s_{i, 2}^{\mathrm{T}}\left(s_{i, 3}+z_{i, 3}\right) \\
& +\sigma_{i, 1} \tilde{W}_{i, 1}^{\mathrm{T}} \hat{W}_{i, 1}+\sigma_{i, 2} \tilde{W}_{i, 2}^{\mathrm{T}} \hat{W}_{i, 2}+\sum_{j=1}^{N} a_{i, j} \varrho_{j, 1} \tilde{W}_{j, 1}^{\mathrm{T}} \hat{W}_{j, 1}+\rho_{i, 2}-\frac{1}{\tau_{i, 2}} z_{i, 2}^{2}+z_{i, 2} M_{i, 2}(\cdot)
\end{aligned}
$$

where $\rho_{i, 2}=\rho_{i, 1}+\frac{1}{2}\left\|\eta_{i, 2}^{0}\right\|^{2}$.

Step $j(3 \leq j<n)$ : Similar to the derivation in Step 2, from (23), we can obtain

$$
\dot{s}_{i, j}=s_{i, j+1}+z_{i, j+1}+\alpha_{i, j}-k_{i, j} \hat{x}_{i, 1}+k_{i, j} x_{i, 1}+\hat{W}_{i, j}^{\mathrm{T}} S_{i, j}\left(\hat{\bar{x}}_{i, j}\right)-\dot{\nu}_{i, j}
$$


Choose the Lyapunov function candidate as

$$
V_{i, j}=V_{i, j-1}+\frac{1}{2} s_{i, j}^{2}+\frac{1}{2} z_{i, j}^{2}+\frac{1}{2 \gamma_{i, j}} \tilde{W}_{i, j}^{\mathrm{T}} \tilde{W}_{i, j}
$$

where $\gamma_{i, j}$ is a positive design constant.

The time derivative of $V_{i, j}$ along (42) and (43) is

$$
\begin{aligned}
\dot{V}_{i, j} & \leq-l_{\text {min }}\left\|e_{i}\right\|^{2}+s_{i, 1}^{\mathrm{T}} \pi_{i} d_{i} z_{i, 2}-\sum_{k=1}^{j-1} c_{i, k} s_{i, k}^{2}+\sum_{k=1}^{j-1} \sigma_{i, k} \tilde{W}_{i, k}^{\mathrm{T}} \hat{W}_{i, k}+\sum_{j=1}^{N} a_{i, j} \varrho_{j, 1} \tilde{W}_{j, 1}^{\mathrm{T}} \hat{W}_{j, 1} \\
& +s_{i, j-1} s_{i, j}+\sum_{k=2}^{j-1} s_{i, k} z_{i, k+1}-\sum_{k=1}^{j-2}\left(\frac{z_{i, k+1}^{2}}{\tau_{i, k+1}}-z_{i, k+1} M_{i, k+1}(\cdot)\right)+\rho_{i, j-1}+\frac{1}{2}\left\|\eta_{i, j}^{0}\right\|^{2} \\
& +s_{i, j}\left(s_{i, j+1}+z_{i, j+1}+\alpha_{i, j}-k_{i, j} \hat{x}_{i, 1}+k_{i, j} x_{i, 1}+\hat{W}_{i, j}^{\mathrm{T}} S_{i, j}\left(\hat{\bar{x}}_{i, j}\right)+\frac{1}{2} s_{i, j}+\tilde{W}_{i, j}^{\mathrm{T}} S_{i, j}\left(\hat{\bar{x}}_{i, j}\right)-\dot{\nu}_{i, j}\right) \\
& -\frac{1}{\tau_{i, j}} z_{i, j}^{2}+z_{i, j} M_{i, j}(\cdot)-\frac{1}{\gamma_{i, j}} \tilde{W}_{i, j}^{\mathrm{T}} \dot{\hat{W}}_{i, j}
\end{aligned}
$$

Construct the virtual control function $\alpha_{i, j}$ and the parameter adaptive law $W_{i, j}$ as:

$$
\begin{aligned}
\alpha_{i, j} & =-c_{i, j} s_{i, j}-s_{i, j-1}-\frac{1}{2} s_{i, j}+k_{i, j} \hat{x}_{i, 1}-k_{i, j} x_{i, 1}-\hat{W}_{i, j}^{\mathrm{T}} S_{i, j}\left(\hat{\bar{x}}_{i, j}\right)+\dot{\nu}_{i, j} \\
\dot{\hat{W}}_{i, j} & =\gamma_{i, j}\left(s_{i, j} S_{i, j}\left(\hat{\bar{x}}_{i, j}\right)-\sigma_{i, j} \hat{W}_{i, j}\right)
\end{aligned}
$$

where $c_{i, j}>0$ and $\sigma_{i, j}>0$ are design parameters.

From (45), (46) and (47), one has

$$
\begin{aligned}
\dot{V}_{i, j} & \leq-l_{\text {min }}\left\|e_{i}\right\|^{2}+s_{i, 1}^{\mathrm{T}} \pi_{i} d_{i} z_{i, 2}-\sum_{k=1}^{j} c_{i, k} s_{i, k}^{2}+\sum_{k=1}^{j} \sigma_{i, k} \tilde{W}_{i, k}^{\mathrm{T}} \hat{W}_{i, k}+\sum_{j=1}^{N} a_{i, j} \varrho_{j, 1} \tilde{W}_{j, 1}^{\mathrm{T}} \hat{W}_{j, 1} \\
& +s_{i, j} s_{i, j+1}+\sum_{k=2}^{j} s_{i, k} z_{i, k+1}-\sum_{k=1}^{j-1}\left(\frac{z_{i, k+1}^{2}}{\tau_{i, k+1}}-z_{i, k+1} M_{i, k+1}(\cdot)\right)+\rho_{i, j}
\end{aligned}
$$

where $\rho_{i, j}=\rho_{i, j-1}+\frac{1}{2}\left\|\eta_{i, j}^{0}\right\|^{2}$.

Step $n$ : In the final design step,from (12) and (25), one obtains

$$
\dot{s}_{i, n}=u_{i}-k_{i, n} \hat{x}_{i, 1}+k_{i, n} x_{i, 1}+\hat{W}_{i, n}^{\mathrm{T}} S_{i, n}\left(\hat{\bar{x}}_{i, n}\right)-\dot{\nu}_{i, n}
$$

Choose the Lyapunov function candidate as

$$
V_{i, n}=V_{i, n-1}+\frac{1}{2} s_{i, n}^{2}+\frac{1}{2} z_{i, n}^{2}+\frac{1}{2 \gamma_{i, n}} \tilde{W}_{i, n}^{\mathrm{T}} \tilde{W}_{i, n}
$$

where $\gamma_{i, n}$ is a positive design constant.

The time derivative of $V_{i, n}$ along (48) and (49) is

$$
\begin{aligned}
\dot{V}_{i, n} & \leq-l_{\text {min }}\left\|e_{i}\right\|^{2}+s_{i, 1}^{\mathrm{T}} \pi_{i} d_{i} z_{i, 2}-\sum_{k=1}^{n-1} c_{i, k} s_{i, k}^{2}+\sum_{k=1}^{n-1} \sigma_{i, k} \tilde{W}_{i, k}^{\mathrm{T}} \hat{W}_{i, k}+\sum_{j=1}^{N} a_{i, j} \varrho_{j, 1} \tilde{W}_{j, 1}^{\mathrm{T}} \hat{W}_{j, 1} \\
& +s_{i, n-1} s_{i, n}+\sum_{k=2}^{n-1} s_{i, k} z_{i, k+1}-\sum_{k=1}^{n-1}\left(\frac{z_{i, k+1}^{2}}{\tau_{i, k+1}}-z_{i, k+1} M_{i, k+1}(\cdot)\right)+\rho_{i, n-1}+\frac{1}{2}\left\|\eta_{i, n}^{0}\right\|^{2} \\
& -\frac{1}{\gamma_{i, n}} \tilde{W}_{i, n}^{\mathrm{T}} \dot{\hat{W}}_{i, n}+s_{i, n}\left(u_{i}-k_{i, n} \hat{x}_{i, 1}+k_{i, n} x_{i, 1}+\hat{W}_{i, n}^{\mathrm{T}} S_{i, n}\left(\hat{\bar{x}}_{i, n}\right)+\tilde{W}_{i, n}^{\mathrm{T}} S_{i, n}\left(\hat{\bar{x}}_{i, n}\right)+\frac{1}{2} s_{i, n}-\dot{\nu}_{i, n}\right)
\end{aligned}
$$


Construct the virtual control function $\alpha_{i, n}$ and the parameter adaptive law $W_{i, n}$ as:

$$
\begin{aligned}
\alpha_{i, n} & =-c_{i, n} s_{i, n}-s_{i, n-1}-\frac{1}{2} s_{i, n}+k_{i, n} \hat{x}_{i, 1}-k_{i, n} x_{i, 1}-\hat{W}_{i, n}^{\mathrm{T}} S_{i, n}\left(\hat{\bar{x}}_{i, n}\right)+\dot{\nu}_{i, n} \\
\dot{\hat{W}}_{i, n} & =\gamma_{i, n}\left(s_{i, n} S_{i, n}\left(\hat{\bar{x}}_{i, n}\right)-\sigma_{i, n} \hat{W}_{i, n}\right)
\end{aligned}
$$

where $c_{i, n}>0$ and $\sigma_{i, n}>0$ are design parameters.

From (12) and (16), one has

$$
u_{i}(t)=\left\{\begin{array}{c}
-\frac{1}{1+\delta_{i, 1}(t) \varepsilon_{i}}\left(1+\varepsilon_{i}\right)\left[\alpha_{i, n} \tanh \left(\frac{s_{i, n} \alpha_{i, n}}{\epsilon}\right)+\bar{a} \tanh \left(\frac{s_{i, n} \bar{a}}{\epsilon}\right)\right]-\frac{\delta_{i, 2}(t) a}{1+\delta_{i, 1}(t) \varepsilon_{i}}, \\
\varepsilon_{i}\left|u_{i}(t)\right|+a \geq m_{i} \\
\alpha_{i, n}-\bar{m}_{i} \tanh \left(\frac{s_{i, n} \bar{m}_{i}}{\epsilon}\right)-\delta_{i, 3}(t) m_{i}, \\
\varepsilon_{i}\left|u_{i}(t)\right|+a<m_{i}
\end{array}\right.
$$

Since $\forall a \in \mathbb{R}$ and $\epsilon>0,-\iota \tanh (\iota / \epsilon) \leq 0$, in the interval $\left[t_{k}, t_{k+1}\right)$, from (12) we have $s_{i, n} r_{i} \leq 0$. As $\delta_{i, 1}(t) \in[-1,1], \quad \delta_{i, 2}(t) \in[-1,1]$, we have $s_{i, n} r_{i} /\left(1+\delta_{i, 1}(t) \varepsilon_{i}\right) \leq s_{i, n} r_{i} /\left(1+\varepsilon_{i}\right)$, and $\delta_{i, 2}(t) a /(1+$ $\left.\delta_{i, 1}(t) \varepsilon_{i}\right) \leq a /\left(1-\varepsilon_{i}\right)$.

From (51) - (54), one has

$$
\begin{aligned}
\dot{V}_{i, n} & \leq-l_{\text {min }}\left\|e_{i}\right\|^{2}+s_{i, 1}^{\mathrm{T}} \pi_{i} d_{i} z_{i, 2}-\sum_{k=1}^{n} c_{i, k} s_{i, k}^{2}+\sum_{k=1}^{n} \sigma_{i, k} \tilde{W}_{i, k}^{\mathrm{T}} \hat{W}_{i, k}+\sum_{j=1}^{N} a_{i, j} \varrho_{j, 1} \tilde{W}_{j, 1}^{\mathrm{T}} \hat{W}_{j, 1} \\
& +\sum_{k=2}^{n-1} s_{i, k} z_{i, k+1}-\sum_{k=1}^{n-1}\left(\frac{z_{i, k+1}}{\tau_{i, k+1}}-z_{i, k+1} M_{i, k+1}(\cdot)\right)+\rho_{i, n}
\end{aligned}
$$

with

$$
\rho_{i, n}= \begin{cases}\rho_{i, n-1}+\frac{1}{2}\left\|\eta_{i, n}^{0}\right\|^{2}+0.557 \epsilon & , \quad \varepsilon_{i}\left|u_{i}(t)\right|+a \geq m_{i} \\ \rho_{i, n-1}+\frac{1}{2}\left\|\eta_{i, n}^{0}\right\|^{2}+0.2785 \epsilon & , \quad \varepsilon_{i}\left|u_{i}(t)\right|+a<m_{i}\end{cases}
$$

According to Young's inequality, we have

$$
\begin{aligned}
\tilde{W}_{i, k}^{\mathrm{T}} \hat{W}_{i, k} & \leq-\frac{1}{2} \tilde{W}_{i, k}^{\mathrm{T}} \tilde{W}_{i, k}+\frac{1}{2} W_{i, k}^{*}{ }^{\mathrm{T}} W_{i, k}^{*} \\
\tilde{W}_{j, 1}^{\mathrm{T}} \hat{W}_{j, 1} & \leq-\frac{1}{2} \tilde{W}_{j, 1}^{\mathrm{T}} \tilde{W}_{j, 1}+\frac{1}{2} W_{j, 1}^{*} \mathrm{~T} W_{j, 1}^{*} \\
\pi_{i} d_{i} s_{i, 1}^{\mathrm{T}} z_{i, 2} & \leq \frac{\pi_{i}^{2} d_{i}^{2}}{2} s_{i, 1}^{2}+\frac{1}{2} z_{i, 2}^{2} \\
s_{i, k} z_{i, k+1} & \leq \frac{1}{2} s_{i, k}^{2}+\frac{1}{2} z_{i, k+1}^{2} \\
z_{i, k+1} M_{i, k+1}(\cdot) & \leq \frac{z_{i, k+1}^{2} M_{i, k+1}^{2}(\cdot)}{2 \xi}+\frac{\xi}{2}
\end{aligned}
$$

From (55) - (60), one has

$$
\begin{aligned}
\dot{V}_{i, n} & \leq-l_{\text {min }}\left\|e_{i}\right\|^{2}-c_{i, 1} s_{i, 1}^{2}+\frac{\pi_{i}^{2} d_{i}^{2}}{2} s_{i, 1}^{2}-\sum_{k=2}^{n}\left(c_{i, k}-\frac{1}{2}\right) s_{i, k}^{2}-\sum_{k=1}^{n} \frac{1}{2} \sigma_{i, k} \tilde{W}_{i, k}^{\mathrm{T}} \tilde{W}_{i, k} \\
& -\sum_{j=1}^{N} \frac{1}{2} a_{i, j} \varrho_{j, 1} \tilde{W}_{j, 1}^{\mathrm{T}} \tilde{W}_{j, 1}-\sum_{k=1}^{n-1}\left(\frac{1}{\tau_{i, k+1}}-\frac{M_{i, k+1}^{2}(\cdot)}{2 \xi}-\frac{1}{2}\right) z_{i, k+1}^{2}+\rho_{i}
\end{aligned}
$$

where $\rho_{i}=\rho_{i, n}+\frac{\xi}{2}(n-1)+\sum_{k=1}^{n} \frac{1}{2} \sigma_{i, k} W_{i, k}^{*}{ }^{\mathrm{T}} W_{i, k}^{*}+\sum_{j=1}^{N} \frac{1}{2} a_{i, j} \varrho_{j, 1} W_{j, 1}^{*}{ }^{\mathrm{T}} W_{j, 1}^{*}$.

Based on the proposed event-triggered control, the main results can be concluded as follows.

Theorem 1: Consider the nonlinear MASs (1) in the fixed directed communication topology with prescribed performance and unmeasured states with the adaptive updating laws $(31)(31)(41)(47)(53)$ 
and proposed adaptive controller (30)(40)(46)(52)(54) subject to triggered signal (13)(14). Based on Assumption 1-3, all signals of the closed-loop system are SGUUB and the containment control objective is achieved with the containment errors converge to a small residual set nearing the origin with prescribed performance. Moreover, the Zeno behavior is thoroughly excluded.

Proof: Take into account the Lyapunov function candidate for the whole system as

$$
V=\sum_{i=1}^{N} V_{i, n}
$$

The time derivative of $V$ is

$$
\begin{aligned}
\dot{V} & \leq \sum_{i=1}^{N}\left\{-l_{m i n}\left\|e_{i}\right\|^{2}-c_{i, 1} s_{i, 1}^{2}+\frac{\pi_{i}^{2} d_{i}^{2}}{2} s_{i, 1}^{2}-\sum_{k=2}^{n}\left(c_{i, k}-\frac{1}{2}\right) s_{i, k}^{2}-\sum_{k=1}^{n} \frac{1}{2} \sigma_{i, k} \tilde{W}_{i, k}^{\mathrm{T}} \tilde{W}_{i, k}\right. \\
& \left.-\sum_{j=1}^{N} \frac{1}{2} a_{i, j} \varrho_{j, 1} \tilde{W}_{j, 1}^{\mathrm{T}} \tilde{W}_{j, 1}-\sum_{k=1}^{n-1}\left(\frac{1}{\tau_{i, k+1}}-\frac{M_{i, k+1}^{2}(\cdot)}{2 \xi}-\frac{1}{2}\right) z_{i, k+1}^{2}+\rho_{i}\right\} \\
& \leq-\kappa V+R
\end{aligned}
$$

where

$$
\begin{aligned}
\kappa & =\min \left\{l_{\min }, 2\left(c_{i, 1}-\frac{\pi_{i}^{2} d_{i}^{2}}{2}\right), 2\left(c_{i, j}-\frac{1}{2}\right), \frac{\gamma_{i, p}}{\sigma_{i, p}}, \frac{\chi_{j, 1}}{\varrho_{j, 1}}, 2\left(\frac{1}{\tau_{i, k+1}}-\frac{M_{i, k+1}^{2}(\cdot)}{2 \xi}-\frac{1}{2}\right)\right\}, \\
i & =1, \ldots, N, j=2, \ldots, n, p=1, \ldots, n, k=1, \ldots, n-1 . \\
R & =\sum_{i=1}^{N} \rho_{i}
\end{aligned}
$$

Integrating (63) over $[0, \mathrm{t})$, it is easy to obtain that

$$
V(t) \leq e^{-\kappa t} V(0)+\frac{R}{\kappa}\left[1-e^{-\kappa t}\right]
$$

Using $\frac{\left\|s_{i, 1}\right\|^{2}}{2} \leq V$, we have $\left\|s_{i, 1}\right\|^{2} \leq 2 e^{-\kappa t} V(0)+\frac{2 R}{\kappa}\left[1-e^{-\kappa t}\right]$. Therefore, the error surfaces $\left\|s_{i, 1}\right\|$ converge to the compact set $\Omega=\left\{s_{i, 1} \mid\left\|s_{i, 1}\right\| \leq \sqrt{\frac{2 R}{\kappa}}\right\}$, which means $s_{i, 1}$ is bounded, where $s_{i, 1}=\left[s_{1,1}, \ldots, s_{N, 1}\right]^{\mathrm{T}}$. In view of Lemma 3 , we can concluded that the containment errors are bounded with prescribed performance. According to Assumption 2 and 3, it can be obtained that all signals of the closed-loop system are SGUUB.

Furthermore, from $\tau_{i}(t)=r_{i}(t)-u_{i}(t)$, we have $\frac{d\left|\tau_{i}(t)\right|}{d t}=\operatorname{sign}\left(\tau_{i}(t)\right) \dot{\tau}_{i}(t) \leq\left|\dot{r}_{i}(t)\right|$. According to (12), it is known that $\dot{r}_{i}(t)$ is bounded which means there exists positive constant $H_{i}$ with $\dot{r}_{i}(t) \leq$ $H_{i}$. From $\tau\left(t_{i}^{k}\right)=0$, and $\lim _{t_{i} \rightarrow t_{i}^{k+1}} \tau\left(t_{i}\right)=m_{i}, k \in z^{+}$, one can obtain that there exists $t^{*}$, and $t_{i}^{k+1}-t_{i}^{k} \geq t^{*} \geq m_{i} / H_{i}>0$. Therefore, Zeno behavior is avoided.

The proof is completed.

\section{Simulation Results}

To validate the effectiveness of the proposed containment control method, an example of three followers and two leaders is utilized, and the fixed communication graph $\mathcal{G}$ is depicted in Fig.1. The dynamics of three followers are modeled as

$$
\left\{\begin{aligned}
\dot{x}_{i, 1} & =x_{i, 2}+\frac{1}{4} x_{i, 1} \sin \left(x_{i, 1}\right) \\
\dot{x}_{i, 2} & =u_{i}+x_{i, 2} \sin \left(x_{i, 1}\right) \quad i=1,2,3 \\
y_{i} & =x_{i, 1}
\end{aligned}\right.
$$


The dynamics of the leaders are described as

$$
\left\{\begin{array}{l}
y_{4 d}=\sin (t+1.86)-0.6 \\
y_{5 d}=\sin (t+1.86)+0.6
\end{array}\right.
$$

The prescribed performance function is $\omega_{i}(t)=(4.5-1.2) e^{-1 * t}+1.2$. Choose correlative design parameters as $k_{1,1}=k_{2,1}=k_{3,1}=15, k_{1,2}=k_{2,2}=13, k_{3,2}=14, \gamma_{1,1}=1.6, \gamma_{1,2}=8, \gamma_{2,1}=$ $3.3, \gamma_{2,2}=8, \gamma_{3,1}=1.6, \gamma_{3,2}=8, \sigma_{1,1}=\sigma_{1,2}=\sigma_{2,1}=\sigma_{2,2}=\sigma_{3,1}=\sigma_{3,2}=3, \chi_{1,1}=\chi_{2,1}=$ $\chi_{3,1}=8, \varrho_{1,1}=\varrho_{2,1}=\varrho_{3,1}=3, \eta_{1 \min }=\eta_{2 \min }=\eta_{3 \min }=0.6, \eta_{1 \max }=\eta_{2 \max }=\eta_{3 \max }=0.9, \tau=$ $0.02, c_{1,1}=16, c_{2,1}=16, c_{3,1}=15, c_{1,2}=110, c_{2,2}=100, c_{3,2}=30, k_{1,2}=288, k_{2,2}=268, k_{3,2}=$ $127, m_{1}=m_{2}=m_{3}=0.8, \bar{m}_{1}=\bar{m}_{2}=\bar{m}_{3}=1, a=0.1, \bar{a}=0.5, \epsilon=8, \varepsilon_{1}=0.5, \varepsilon_{2}=0.4, \varepsilon_{3}=0.5$.

Choose initial conditions as $x_{1,1}(0)=0.5, x_{2,1}(0)=0.5, x_{3,1}(0)=0.4, x_{1,2}(0)=0.5, x_{2,2}(0)=$ $0.5, x_{3,2}(0)=0.4, \hat{x}_{1,1}(0)=0.5, \hat{x}_{2,1}(0)=0.5, \hat{x}_{3,1}(0)=0.4$.

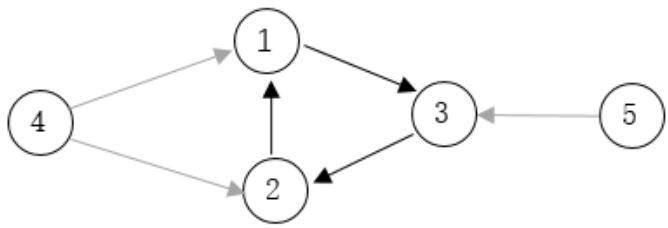

Fig. 1: Communication graph.

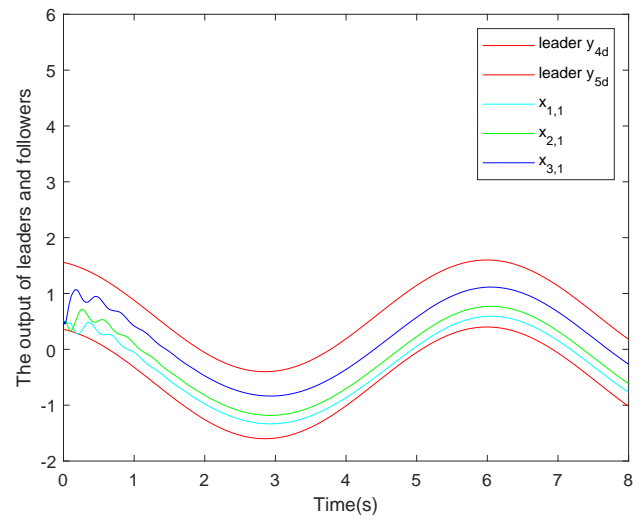

Fig. 2: Trajectories of $x_{i, 1}(i=1,2,3)$ and leaders $y_{4 d}$ and $y_{5 d}$.

Fig. 2-7 describe the simulation results. Fig. 2 shows the containment result that the outputs of all followers asymptotically converge to the convex hull formed by the two leaders. Fig. 3 plots the second-order states of three followers. Fig. 4 presents the states $x_{i, 1}$ and $x_{i, 2}(i=1,2,3)$ and their corresponding observers, and it is shown that the system outputs can be well estimated by NN-based observer. The containment errors and performance bounds are shown in Fig. 5, which indicate the control schemes can impose $v_{i, 1}$ to remain within the prescribed bounds. Fig. 6 shows the trajectories of the event-triggered control signals $u_{i}$. The event-triggered times for three followers are expressed in Fig. 7. From the simulation results, the efficiency of the proposed control scheme can be verified. 


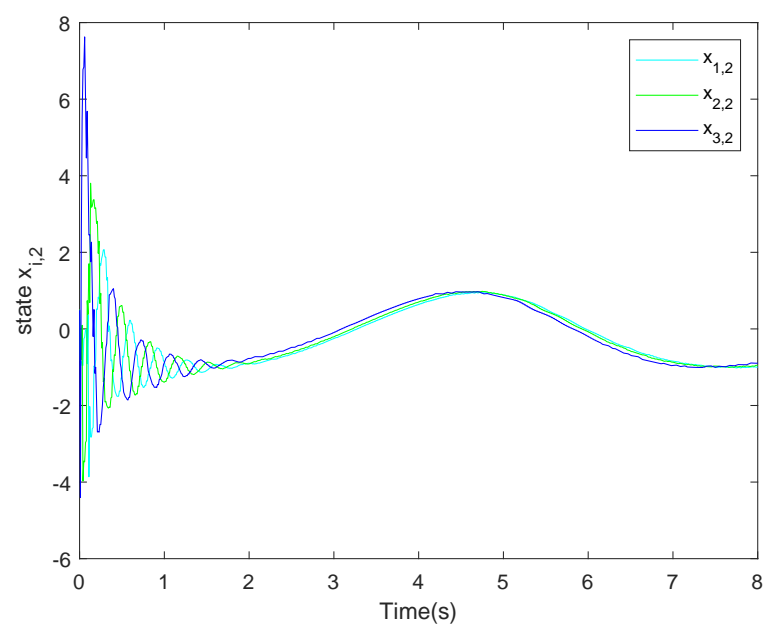

Fig. 3: Trajectories of $x_{i, 2}(i=1,2,3)$.
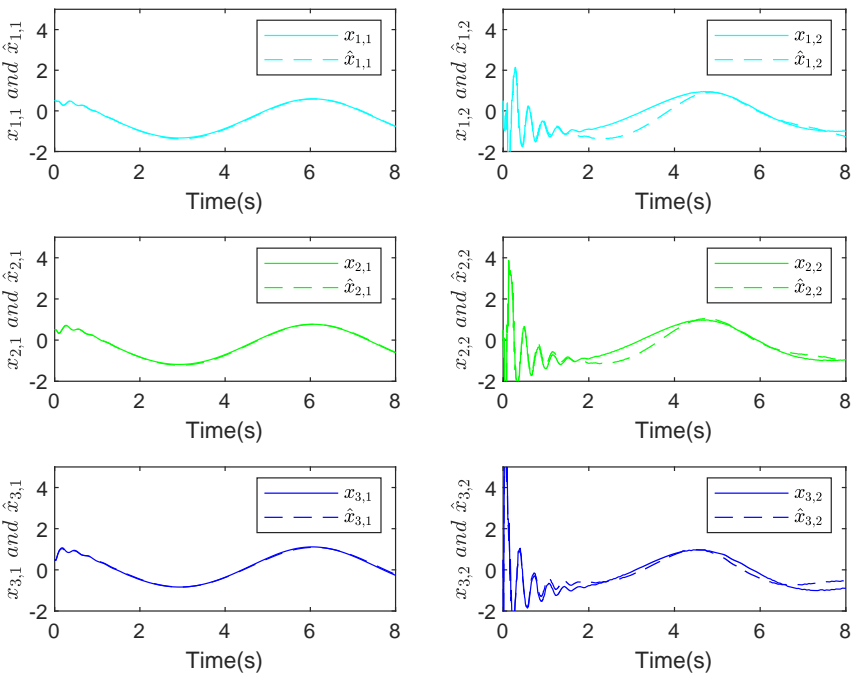

Fig. 4: States and their observers.

\section{Conclusion}

In this paper, the adaptive event-triggered containment control scheme has been investigated for a class of strict-feedback nonlinear MASs with prescribed performance and unmeasured states which can be estimated by NN-based observers. Based on the backstepping control and DSC technique, the adaptive consensus controllers are developed. It is proved that the containment control purpose has been accomplished. Especially, the control signals have been updated by switching threshold eventtriggered mechanism, which provides more elasticity in the balance of network constraints and system performance. On account of the prescribed performance function, the local neighborhood containment errors converge into a prescribed set. According to the Lyapunov stability theory, it is demonstrated that all signals of the closed-loop system are SGUUB. Finally, the effectiveness of the proposed containment control approach is confirmed by simulation example. 


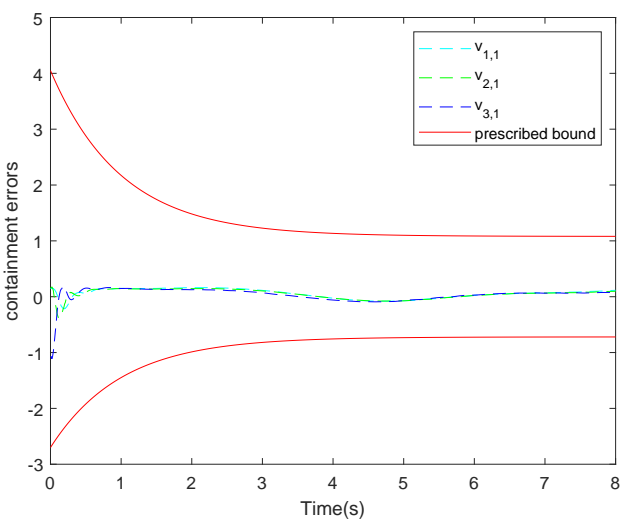

Fig. 5: Curves of containment errors $v_{i, 1}(i=1,2,3)$ and performance bounds.

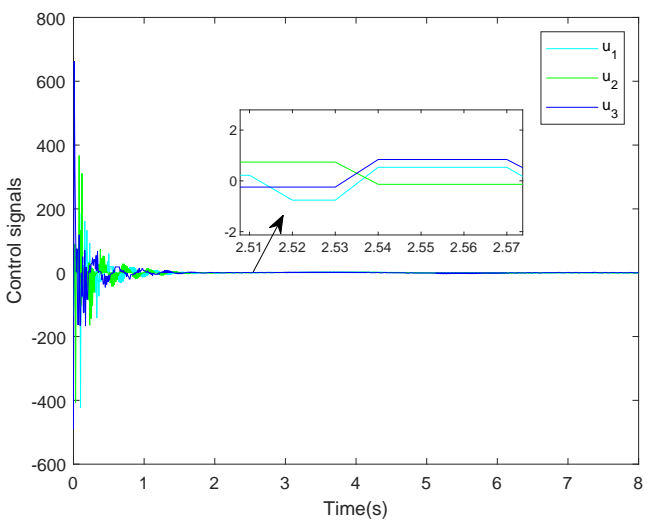

Fig. 6: Control signals $u_{i}(i=1,2,3)$.

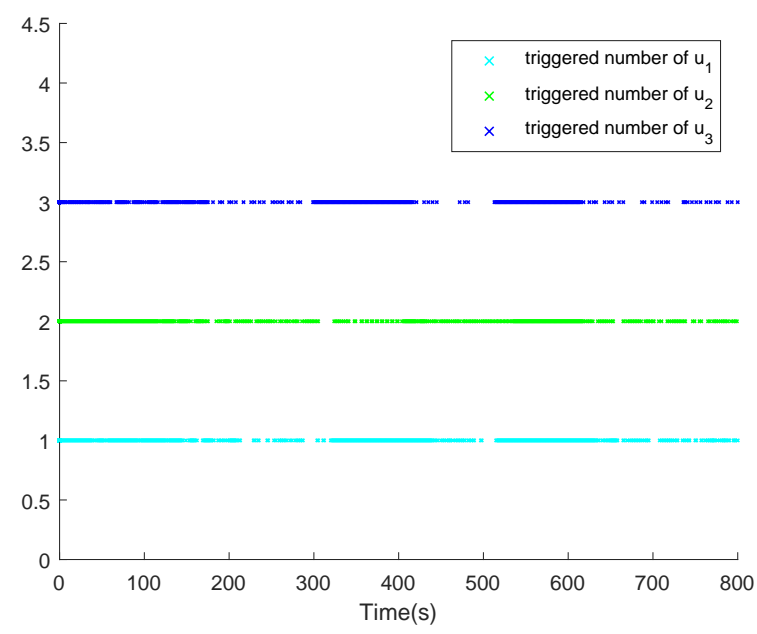

Fig. 7: The triggered number under the switching threshold strategy. 


\section{Acknowledgements}

This work was supported by Natural Science Foundation Project of Chongqing under Grant cstc2019jcyj$\operatorname{msxmX036}$

\section{Compliance with ethical standards}

Funding

This work was supported by by Natural Science Foundation Project of Chongqing under Grant cstc2019jcyj-msxmX036.

Conflict of interest

The authors declare that they have no conflict of interest.

Ethical approval

This article does not contain any experiments with human or animal participants performed by any of the authors.

Informed consent

Informed consent was obtained from all individual participants included in the study.

\section{References}

1. P. Ogren, E. Fiorelli, and N.E. Leonard. Cooperative control of mobile sensor networks:adaptive gradient climbing in a distributed environment. IEEE Transactions on Automatic Control, 49(8):1292-1302, 2004.

2. Lin Zhao, Jinpeng Yu, and Peng Shi. Command filtered backstepping-based attitude containment control for spacecraft formation. IEEE Transactions on Systems, Man, and Cybernetics: Systems, 51(2):1278-1287, 2021.

3. Zhouhua Peng, Jun Wang, and Dan Wang. Containment maneuvering of marine surface vehicles with multiple parameterized paths via spatial-temporal decoupling. IEEE/ASME Transactions on Mechatronics, 22(2):10261036, 2017.

4. Zhouhua Peng, Dan Wang, Yang Shi, Hao Wang, and Wei Wang. Containment control of networked autonomous underwater vehicles with model uncertainty and ocean disturbances guided by multiple leaders. Information Sciences, 316:163-179, 2015. Nature-Inspired Algorithms for Large Scale Global Optimization.

5. Xiwang Dong, Bocheng Yu, Zongying Shi, and Yisheng Zhong. Time-varying formation control for unmanned aerial vehicles: Theories and applications. IEEE Transactions on Control Systems Technology, 23(1):340-348, 2015

6. Jie Mei, Wei Ren, and Guangfu Ma. Distributed containment control for lagrangian networks with parametric uncertainties under a directed graph. Automatica, 48(4):653-659, 2012.

7. Yiguang Hong, Jiangping Hu, and Linxin Gao. Tracking control for multi-agent consensus with an active leader and variable topology. Automatica, 42(7):1177-1182, 2006.

8. M. Ji, G. Ferrari-Trecate, M. Egerstedt, and A. Buffa. Containment control in mobile networks. IEEE Transactions on Automatic Control, 53(8):1972-1975, 2008.

9. Z. Li, G. Wen, Z. Duan, and W. Ren. Designing fully distributed consensus protocols for linear multi-agent systems with directed graphs. IEEE Transactions on Automatic Control, 60(4):1152-1157, 2015.

10. Z. Li, R. Wei, X. Liu, and M. Fu. Distributed containment control of multi-agent systems with general linear dynamics in the presence of multiple leaders. International Journal of Robust and Nonlinear Control, 23(5), 2013

11. Hamed Haghshenas, Mohammad Ali Badamchizadeh, and Mahdi Baradarannia. Containment control of heterogeneous linear multi-agent systems. Automatica, 54:210-216, 2015.

12. M. Jie, R. Wei, L. Bing, and G. Ma. Distributed containment control for multiple unknown second-order nonlinear systems with application to networked lagrangian systems. IEEE Transactions on Neural Networks and Learning Systems, 26(9), 2015

13. Yoo and Sung Jin. Distributed adaptive containment control of uncertain nonlinear multi-agent systems in strict-feedback form. Automatica, 49(7):2145-2153, 2013.

14. W. Wang and S. Tong. Adaptive fuzzy containment control of nonlinear strict-feedback systems with full state constraints. IEEE Transactions on Fuzzy Systems, 27(10):2024-2038, 2019.

15. Shaocheng Tong and Yongming Li. Observer-based fuzzy adaptive control for strict-feedback nonlinear systems. Fuzzy Sets and Systems, 160(12):1749-1764, 2009. Theme: Control Engineering. 
16. S. C. Tong, Y. M. Li, F. Gang, and T. S. Li. Observer-based adaptive fuzzy backstepping dynamic surface control for a class of mimo nonlinear systems. IEEE Transactions on Systems Man and Cybernetics Part B, 41(4):0-1135, 2011

17. Y. Li, S. Tong, L. Liu, and G. Feng. Adaptive output-feedback control design with prescribed performance for switched nonlinear systems. Automatica, 80:225-231, 2017.

18. C. P. Bechlioulis and G. A. Rovithakis. Robust adaptive control of feedback linearizable mimo nonlinear systems with prescribed performance. IEEE Transactions on Automatic Control, 53(9):2090-2099, 2008.

19. Y. Li and S. Tong. Prescribed performance adaptive fuzzy output-feedback dynamic surface control for nonlinear large-scale systems with time delay. Information Sciences, 292:125-142, 2015

20. C. P. Bechlioulis and G. A. Rovithakis. A low-complexity global approximation-free control scheme with prescribed performance for unknown pure feedback systems. Automatica, 50(4):1217-1226, 2014.

21. J. Ni, C. K. Ahn, L. Liu, and C. Liu. Prescribed performance fixed-time recurrent neural network control for uncertain nonlinear systems. Neurocomputing, 363(Oct.21):351-365, 2019.

22. H. Liang, Y. Zhang, T. Huang, and H. Ma. Prescribed performance cooperative control for multiagent systems with input quantization. IEEE Transactions on Cybernetics, pages 1-10, 2019.

23. Wei Wang, Hongjing Liang, Yingnan Pan, and Tieshan Li. Prescribed performance adaptive fuzzy containment control for nonlinear multiagent systems using disturbance observer. IEEE Transactions on Cybernetics, 50(9):3879-3891, 2020.

24. Tong Wang, Yifu Zhang, Jianbin Qiu, and Huijun Gao. Adaptive fuzzy backstepping control for a class of nonlinear systems with sampled and delayed measurements. IEEE Transactions on Fuzzy Systems, 23(2):302312,2015

25. Lantao Xing, Changyun Wen, Zhitao Liu, Hongye Su, and Jianping Cai. Event-triggered adaptive control for a class of uncertain nonlinear systems. IEEE Transactions on Automatic Control, 62(4):2071-2076, 2017.

26. Eloy Garcia and Panos J. Antsaklis. Model-based event-triggered control with time-varying network delays. In 2011 50th IEEE Conference on Decision and Control and European Control Conference, pages 1650-1655, 2011.

27. Paulo Tabuada. Event-triggered real-time scheduling of stabilizing control tasks. IEEE Transactions on Automatic Control, 52(9):1680-1685, 2007.

28. L. Xing, C. Wen, Z. Liu, H. Su, and J. Cai. Event-triggered output feedback control for a class of uncertain nonlinear systems. IEEE Transactions on Automatic Control, pages 290-297, 2018.

29. H. Ma, H. Li, H. Liang, and G. Dong. Adaptive fuzzy event-triggered control for stochastic nonlinear systems with full state constraints and actuator faults. IEEE Transactions on Fuzzy Systems, pages 1-1, 2019.

30. Yanhui Zhang, Jian Sun, Hongjing Liang, and Hongyi Li. Event-triggered adaptive tracking control for multiagent systems with unknown disturbances. IEEE Transactions on Cybernetics, 50(3):890-901, 2020.

31. Changjiang $\mathrm{Xi}$ and Jiuxiang Dong. Event-triggered adaptive fuzzy distributed tracking control for uncertain nonlinear multi-agent systems. Fuzzy Sets and Systems, 402:35-50, 2021. Theme: Control Engineering.

32. Jianbin Qiu, Kangkang Sun, Tong Wang, and Huijun Gao. Observer-based fuzzy adaptive event-triggered control for pure-feedback nonlinear systems with prescribed performance. IEEE Transactions on Fuzzy Systems, 27(11):2152-2162, 2019.

33. Ziyang Meng, Wei Ren, and Zheng You. Distributed finite-time attitude containment control for multiple rigid bodies. Automatica, 46(12):2092-2099, 2010. 